\title{
A sapphire monolithic differential accelerometer as core sensor for gravity gradiometric geophysical instrumentation
}

\author{
Alessandro Bertolini $\left(\left(^{1}\right)\left({ }^{3}\right)\left({ }^{*}\right)\right.$, Nicolò Beverini $\left({ }^{1}\right)\left({ }^{2}\right)$, Andrea De Michele $\left({ }^{1}\right)$, \\ Francesco Fidecaro $\left({ }^{1}\right)$ and Francesco Mango $\left({ }^{1}\right)$ \\ ( $\left.{ }^{1}\right)$ Dipartimento di Fisica «E. Fermi», Università degli Studi di Pisa, \\ and Istituto Nazionale di Fisica della Materia (INFM), CNR, Pisa, Italy \\ $\left(^{2}\right)$ Consorzio Universitario della Spezia, La Spezia, Italy \\ ( $\left.{ }^{3}\right)$ Marwan Technology Srl Pisa, Italy
}

\begin{abstract}
Gradiometric gravimetry is a survey technique widely used in geological structure investigation. This work demonstrates the feasibility of a new class of low frequency accelerometers for geodynamics studies and space applications. We present the design features of a new low noise single-axis differential accelerometer; the sensor is suitable to be used in a Gravity Gradiometer (GG) system for land geophysical survey and gravity gradient measurements. A resolution of 1 Eötvös $\left(1 \mathrm{EÖ}=10^{-9} \mathrm{~s}^{-2}\right)$ at one sample per second is achievable in a compact, lightweight (less than $2 \mathrm{~kg}$ ) portable instrument, operating at room temperature. The basic components of the sensor are two identical rigidly connected accelerometers separated by a $15-\mathrm{cm}$ baseline vector and the useful signal is extracted as the subtraction of the two outputs, by means of an interferometric microwave readout system. The structure will be engraved in a monocrystal of sapphire by means of Computer-Numerically-Controlled (CNC) ultrasonic machining: the material was chosen because of its unique mix of outstanding mechanical and dielectric properties.
\end{abstract}

Key words gradiometric gravimeter-accelerometer - Whispering gallery cavity

\section{Introduction}

The gravimetric methods are widely used in geological structure investigations, in particular in oil and mine exploration industry. This kind of research requires a very high level of instrumental sensitivity and an accuracy of the order of one

Mailing address: Prof. Nicolò Beverini, Dipartimento di Fisica «E. Fermi», Università degli Studi di Pisa, Largo B. Pontecorvo 3, 56127 Pisa, Italy; e-mail: beverini@df.unipi.it

$\left(^{*}\right)$ Present address: DESY, Notkestrasse 85, D 22607 Hamburg, Germany.
microGal $\left(1 \mathrm{Gal}=1 \mathrm{~cm} / \mathrm{s}^{2}\right)$, that is $10^{-9} \mathrm{~g}$, in order to achieve significant results. At this sensitivity level spurious effects, not connected with underground mass distributions, but that are due to surface topographic irregularities or to anthropic activities or to tidal fluctuations become important. The instrumental drift, unavoidable in mechanical sensors because of creep of the elastic elements, thermal gradients etc., is another source of errors, which are more and more important as the data acquisition time is longer. Much more severe constraints rise in implementing a gravimeter on a ship or on a plane, because the instrument cannot distinguish between gravity and kinematic acceleration vectors.

Gravity gradiometric methods can avoid many of these problems. This technique is based on the measurement of one or more of the five independent components of the tensor 
$\Gamma_{i j}=\partial g_{j} / \partial x_{i}$ (that is the gradient of the gravity vector $\boldsymbol{g}$ ), as the difference between the values of the $g_{j}$ components across a fixed distance basis. This technique has demonstrated his significance in the oil deposit signature and in the research and localization of underground cavities.

The on-field implementation of the gradiometric principle can be made by reading, with a high sensitivity gravimetric instrument, the vertical gravity values $g_{z}$ in two different point, separated by a known vectorial basis $r_{i}$, and evaluating the $\Gamma_{i z}$ component of the tensor. However, the full exploitation of the peculiarity of the gradiometric surveys could be achieved by using a single instrument with built-in differential readout capability, in order to efficiently reject common mode noise and spurious signal, like kinematic effects due to linear accelerations of the measurement platform. The basic practical implementation consists of two identical accelerometers fixed rigidly together with a common sensitive axis (see fig. 1).

Below the mechanical resonant frequency $\omega_{0}$ of the accelerometers, the difference in the

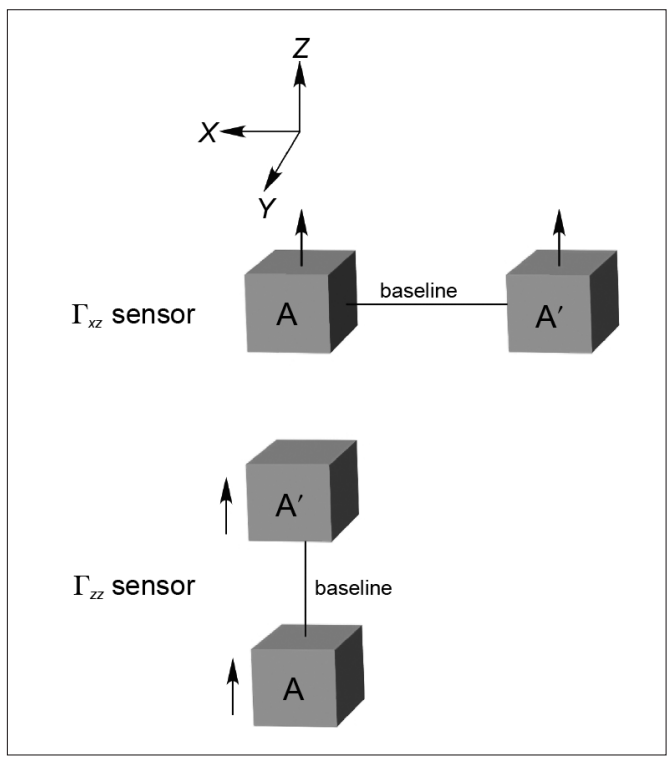

Fig. 1. Example basic configurations of a singlecomponent gravity gradiometer. The geometric arrangement of the two linear accelerometers is shown. vertical position of the proof masses is linearly related to the gravity gradient component $\Gamma_{i z}$ according to

$$
z_{1}-z_{2} \approx \frac{\Gamma_{i z} r_{i}}{\omega_{0}^{2}}+2\left(\frac{\delta \omega}{\omega_{0}}\right) \frac{\left(g_{z}+a_{z}\right)}{\omega_{0}^{2}} .
$$

The second term is related to static mechanical mismatch $\delta \omega$ between the two accelerometers. Being almost impossible to build two perfectly matched accelerometers, a residual sensitivity to kinematic effects arises, polluting the signal channel by an amount proportional to the platform motion acceleration $a_{z}$. The factor $\omega_{0} / 2 \delta \omega$ is defined as the Common-Mode-Rejection-Ratio (CMRR). Detecting 1 Eö gravity gradient in presence of $10^{-3} \mathrm{~m} / \mathrm{s}^{2}$ linear acceleration, with an instrument with $15-\mathrm{cm}$ baseline, requires a 130 $\mathrm{dB}$ of CMRR. Non-linearities of the accelerometer spring system also affect the CMRR for large environmental accelerations. This imposes suitable vibration isolation platforms, especially in airborne instruments.

At present only a few gravity gradiometers have been developed. Spaceborne gravity gradiometer devices are capable of outstanding noise levels $\left(10^{-4}-10^{-3}\right.$ Eö) but rely on the very low environmental noise of a low acceleration satellite (at least one million times more silent than a typical light aircraft, for instance). Two examples are the 6-axis superconducting differential accelerometer developed by Ho Jung Paik group at University of Maryland (Chain and Paik, 1988a,b), and now being built by Oxford Instruments, and the GOCE Mission accelerometer (Rebhan et al., 2000). Laboratory use only apparatus have also been devised by doubling the core sensor of absolute gravimeters, like the superconducting levitation device from GWR Inc. (Richter and Warburton, 1998), the free-fall Michelson interferometer from Micro- $g$ Solutions Inc. (http://www.microgsolutions.com/gradiometer.pr ess.htm) and the atom interferometer developed by Kasevich group at Yale University (Snadden et al., 1998). All these instruments claim a resolution ranging from 0.1 to 1 Eö at one sample per second, but they are still far away from the portability and the on-field applications.

The most popular portable instrumentation is the Gravity Gradiometer Instrument (GGI), de- 


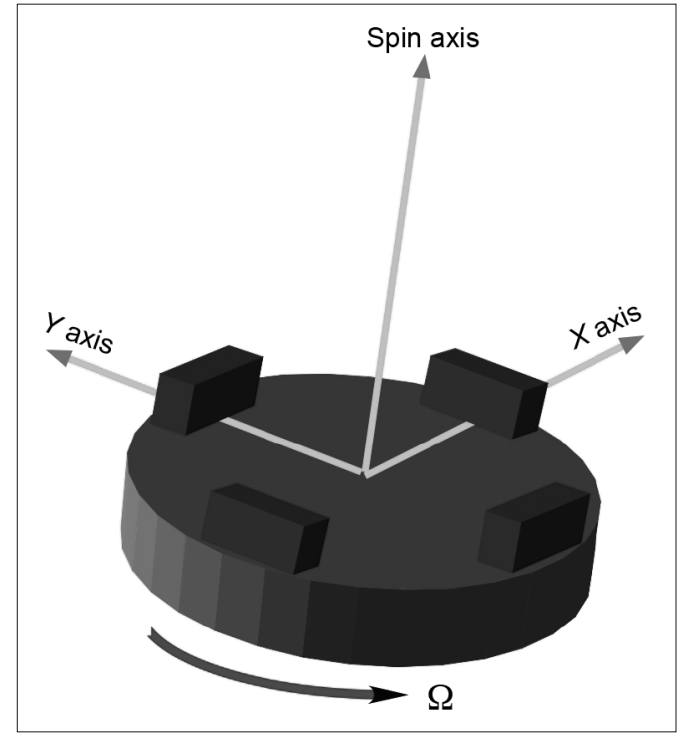

Fig. 2. Basic scheme of a GGI (Gravity Gradiometer Instrument): two opposite matched pairs of linear accelerometers sit on a spinning platform. The gravity gradient signal is given by a linear combination of the accelerometer outputs at twice the spinning frequency.

veloped primarily by Lockheed Martin for military application, but recently declassified and presented now on the civil market (Goldstein and Brett, 1998). They are instruments specifically designed to be mounted on mobile platforms (aircrafts or ships). In the simplest form it consists of two matched pairs of force-balance accelerometers mounted orthogonally on a rotating platform (see fig. 2). Linear accelerations perpendicular to the rotation axis are modulated at the spinning frequency, while the gravity gradient effect is modulated at twice spinning frequency. In this way the useful signal is separated in the frequency domain from the instrument bias and vehicle accelerations (e.g., reference ellipsoid, latitude, earth tide and isostatic effects). The signal is extracted by a suitable combination of the difference in readings between opposing pairs of accelerometers. A high CMRR is achieved by a feedback system acting on the accelerometers scale-factor, and by the effect of the rotating platform. The sensitivity declared is of the order of 5
Eö. The GGI is the basic element of the most complete instrument, the Bell Geospace Full Tensor Gradiometer (http://www.bellgeo.com/ tech/system.html). This instrument measures at the same time all the components of the gradient tensor, allowing a complete reconstruction of the gravity map. The performances of this instrument are very high; it is however a high cost cumbersome instrument, of large volume and weight that must be carried on a ship or a plane. More recently the Bell technology has been shared for advanced developments, the Falcon Gravity Gradiometer project, with the Australian company BHP Billiton Discovery Technologies (Lee, 2001). The Falcon system has proven to be effective in detecting sources with $\Gamma_{z z}>10$ Eö rms and a full width at half maximum of $500 \mathrm{~m}$ of spatial resolution. At this time, the most promising portable instrument seems to be the mobile superconducting beam-balance gravity gradiometer developed by F. Van Kann (Van Kann et al., 1994) at University of Western Australia and now being launched on the market by Gedex Inc. (for details see Matthews, 2002). The instrument is specifically designed for airborne surveys and is capable of unprecedented CMRR (exceeding 190 $\mathrm{dB}$ ) and of a resolution better than 1 Eö at one sample per second, even in the presence of $1 \mathrm{~m} / \mathrm{s}^{2}$ linear accelerations. However, even in this case, the size of the accessory inertial platform and of the liquid helium cooling system still limit the portability of the instrument.

No available device currently fills the gap between laboratory-only apparatus and large size, costly GGs for airborne and shipborne applications. In this work we present the design of a new core sensor for an advanced lightweight portable gravity gradiometer.

The sensor is a differential vertical accelerometer using state-of-art dielectric crystals machining techniques and microwave position sensing technologies to achieve 1 Eö level resolutions operating even at room temperature.

\section{The mechanical apparatus}

The instrument consists of three main parts: the accelerometer plate, hosting two identical accelerometers sensitive along the $z$-axis, the refer- 
ence plate, hosting the motion sensing microwave elements and a conductive shield. Both the accelerometer and the reference plates are completely carved out of a high quality singlecrystal sapphire plate $1-\mathrm{cm}$ thick by means of Computer Numerically Controlled (CNC) chisel ultrasonic machining (Bertolini et al., 2003). Ultrasonic machining yields complex shapes in crystals, ceramics and composite materials without any release of thermal and mechanical stress into the work piece. Because of its geometry, in the present apparatus design, the instrument is sensitive to the off-line $\Gamma_{y z}$ component of the gravity gradient tensor. Each accelerometer is a small torsion pendulum with a $0.04 \mathrm{~kg}$ proof mass suspended by two torsion beams. The baseline, defined as the relative position of the centers of mass of the moving bodies (fig. 3), is 15$\mathrm{cm}$. A force $F_{z}$ applied to the proof mass produces a twist $\theta$ of the torsion pendulum given by

$$
\vartheta \approx \frac{3 F_{z} l d}{b t^{3} G}
$$

where $l, b, t$ are respectively the length, height and thickness of the torsion beams; $G$ is the rigidity modulus of the material and $d$ is the dis-

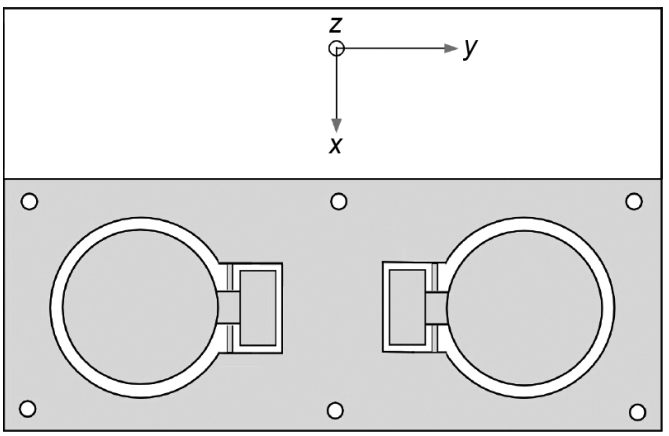

Fig. 3. Top view of the differential accelerometer mechanics. Two identical torsion pendula are carved out of a single-crystal sapphire plate by means of CNC ultrasonic impact grinding. The two disks are used as the tuning elements of two sapphire-loaded microwave resonators. Two flaps with evaporated gold electrodes are used for electrostatic stiffness fine balancing and cold damping of the accelerometers. tance between the center-of-mass and the rotation axis. By using sapphire beams $1 \times 1 \mathrm{~mm}^{2}$ in section and $10 \mathrm{~mm}$ long, a resonant frequency of $20 \mathrm{~Hz}$ can be obtained. In this configuration, a 1 Eö gravity gradient is equivalent to a differential displacement of the GG test masses of $10^{-14} \mathrm{~m}$. Sapphire has been chosen because its excellent mechanical and dielectric properties. It combines high strength, low hysteresis and very low electrical losses; HEMEX ${ }^{\mathrm{TM}}$ grade sapphire single-crystal exhibit mechanical and electrical quality factors greater than $10^{5}$ even at room temperature (http://www.crystalsystems.com). The monolithic structure obtained by CNC machining allows the accuracy in the relative positioning of the different mechanical parts to be kept within a few micrometers. The monolithic design also allows very low hysteresis, eliminating shear effects at the contact surfaces between separate mechanical parts (stick-andslip), while the use of a single material limits the instrument's thermal sensitivity. Co-machining of both the torsion springs effectively strongly reduces directional cross-talks due to misalignments between the components in the assembly, typical of a composite structure. In this way, a $60 \mathrm{~dB}$ mechanical common mode rejection factor can be reasonably achieved just by construction. A fine trimming of the CMRR will be achieved by acting on the «flap» electrodes. By applying a high-voltage on both sides of a flap, a tunable negative stiffness effect is realized. The trimming procedure will make use of a suitable tilting jig which eventually should even be integrated in the GG system to allow for an automated balancing routine. Tilting is the easiest way to inject a $1-\boldsymbol{g}$ level common-mode acceleration into the system and to allow in this way for a high signal-to-noise ratio in the balancing operation. We expect to gain an additional $60 \mathrm{~dB}$ in the instrument's CMRR. The residual sensitivity to environmental linear accelerations will be cancelled out by using a suitable vertical mechanical active isolation system, driven, with a proper feedback gain, by the common-mode acceleration signal itself.

Both the elastic constant of the single oscillator and its inertia moment are affected by temperature. Such thermal effects are not a 
problem, as long as both sensors are at the same temperature. A difference of $1^{\circ} \mathrm{C}$ between the two sensors can however produce a sensitivity difference of the order of $10^{-5}$. In order to achieve 1 Eö sensitivity the temperature differences must be controlled better than $10^{-5} \mathrm{~K}$. It will be necessary an efficient passive isolation of the instrument container. The monolithic structure of the apparatus and the vacuum environment, already necessary in order to avoid viscous damping of the mechanical resonance, can warrant a very high thermal inertia. Semiconductor temperature sensors will monitor the eventual thermal gradient.

\section{The microwave transducer and readout system}

The motion of the proof-mass of each accele-rometer is detected by means of a microwave Sapphire Dielectric Resonator Transducer (SDRT) (Blair et al., 1992). In the simplest form, a SDRT consists of two coaxial disks mounted inside a conducting shell which prevents radiation losses and unwanted coupling with the environment. Each disk resonates with a Whispering Gallery (WG) mode of the electromagnetic field. These modes are generated by total reflection of the electromagnetic field on the external surface of the dielectric cylinder. By coupling two of these resonators we obtain a new resonator whose resonant frequency $f$ is a function of the gap width between the two disks (fig. 4). With a suitable choice of the resonator geometry is possible to optimize the merit factor of the transducer $Q \cdot \mathrm{d} f / \mathrm{d} z$, the product between the quality factor of the WG coupled mode and the tuning coefficient, which determines the displacement sensitivity of the transducer itself.

There are two types of WG modes: $E$ modes $\left(E_{z}, H_{\rho}, H_{\phi}\right)$ and $H$ modes $\left(H_{z}, E_{\rho}, E_{\phi}\right)$, where $z$ denotes the component along the disk rotation axis of the field vector, $\rho$ its radial component, and $\phi$ its azimuthal component. Each mode is labeled by three numbers $m, n$ and $k$, describing the number of field maximums in the azimuth, radial and longitudinal direction respectively. The principal modes $(n=k=1)$ allow $Q$ factors that can be equal or even greater than the in-

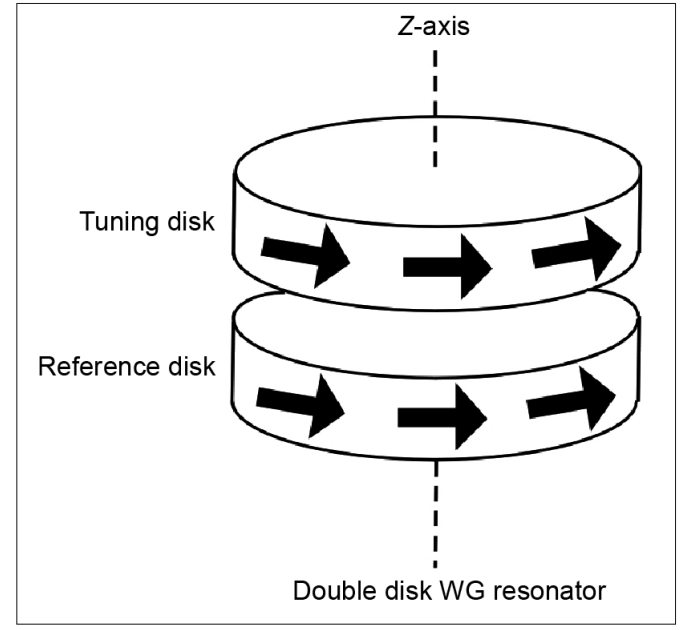

Fig. 4. Sketch of a double-disk Whispering Gallery (WG) microwave resonator.

verse of the dielectric loss tangent of the dielectric material. The most sensitive resonances to changes in the gap spacing has been demonstrated to be the WG $E$ modes, with high order azimuthal number (Blair et al., 1998). The reason is the $E$ modes have a higher field density in the gap between the disks. Because of the continuity condition of the electromagnetic vector at the boundary of a dielectric, the $E_{z}$ component of $E$ mode increases from the value $E_{z \text { (vacuum) }}$ in the gap spacing to $\varepsilon_{z} E_{z \text { (vacuum) }}$ in the dielectric, whereas the main field components of $H$ mode remain unchanged.

The tuning coefficient is also a function of the height of the disks. When the disk thickness decreases, the fraction of the electromagnetic power in the gap increases. As a consequence, the value of $d f / d z$ increases.

In our design the proof mass of each accelerometer is used as the tuning disk of the SDRT formed with the corresponding reference puck dug into the reference plate (see fig. 5). The dimensions of the disks was chosen in the way that the high order azimuthal whispering gallery $E$ modes $(m=7-12)$ resonate in the $X$ band (between 9 and $12 \mathrm{GHz}$ ).

We have performed some preliminary tests on the SDRT using two sapphire disks with $42 \mathrm{~mm}$ 


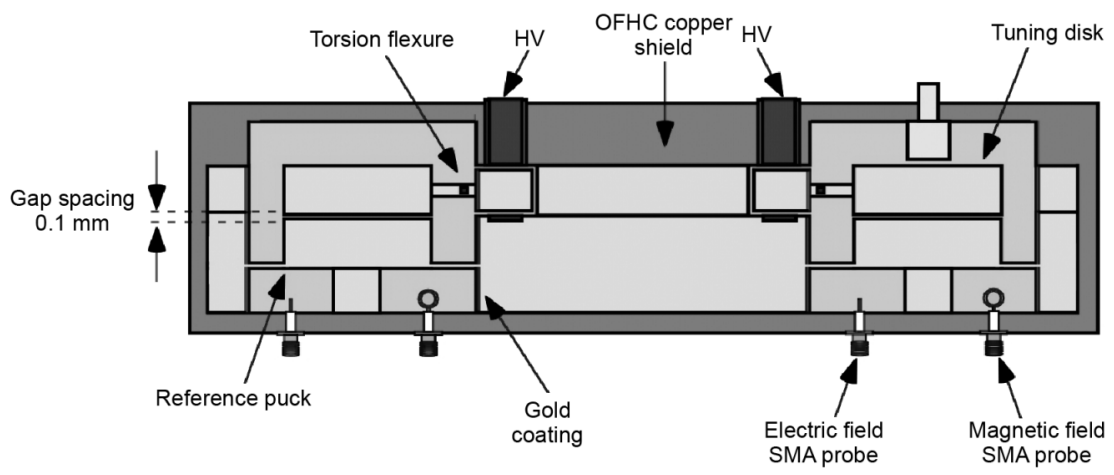

Fig. 5. Section view of the differential accelerometer. The accelerometer and the reference plates are surrounded by a metallic shielding enclosure. The two pairs of control electrodes allow for fine trimming of the CMRR and cold damping of the accelerometer motion.

Table I. Experimental and theoretical resonance frequencies of a Sapphire Dielectric Resonator made of two disks of $42 \mathrm{~mm}$ diameter and $5 \mathrm{~mm}$ height in contact.

\begin{tabular}{cccc}
\hline \hline Mode & Exp $(\mathrm{GHz})$ & Theo $(\mathrm{GHz})$ & $Q$ \\
\hline$E_{7,1,1}$ & 8.235 & 8.262 & 14000 \\
$E_{8,1,1}$ & 8.889 & 8.918 & 18000 \\
$E_{9,1,1}$ & 9.551 & 9.581 & 24000 \\
$E_{10,1,1}$ & 10.221 & 10.250 & 26000 \\
$E_{11,1,1}$ & 10.897 & 10.926 & 55000 \\
$E_{12,1,1}$ & 11.578 & 11.606 & 58000 \\
\hline
\end{tabular}

in diameter and $5 \mathrm{~mm}$ in height. The sapphire crystals were of optical grade quality and without surface polish. The distance between the two disks was changed moving vertically one of them mounted on a micrometer screw. A microwave sweeper was used as tuneable microwave source and a diode as square law detector.

Table I reports the experimental and theoretical value of the resonance frequencies of some modes when the two disks are in contact (zero distance), and the $Q$ factor of the resonances. The theoretical frequencies are calculated using the method described in Blair et al. (1998). Figure 6 plots experimental and theoretical values of resonance frequency and of the tuning coefficient $\mathrm{d} f / \mathrm{d} z$, as a function of the distance between the two disks for the $E_{11,1,1}$ mode. These results demonstrate that a gap spacing of 100 $\mu \mathrm{m}$ ensures for the $E_{11,1,1}$ mode in our geometry a high tuning coefficient, around $6 \mathrm{MHz} / \mu \mathrm{m}$, and a quality factor of the order of $Q \approx 50000$ 60000 even at room temperature. Higher $Q$ values can be expected with sapphire crystals of better quality.

Radiation loss shielding is achieved by gold plating the inner surface of the sapphire plates. The readout system will be realized following the fully differential interferometric scheme introduced by Blair et al. (1994), which has been demonstrated to be capable of displacement resolutions better than $10^{-14} \mathrm{~m}$. The idea is to use one of the two resonators to build a loop oscillator and excite the second one. The differential acceleration is detected by measuring the frequency mismatch between the two SDRT with a balanced detector. A practical implementation is sketched in fig. 7. A stable microwave signal is generated by a loop oscillator including one of the gradiometer resonators as the reference cavity, a phase shifter and a low noise high-gain microwave amplifier. A power divider splits the microwave power between the two resonators used in trasmission mode. Coupling is achieved by means of two circulators. The power transmitted by the SDRTs is compared by means of a $180^{\circ}$ power combiner, whose output is demodulated in-phase with respect to the loop oscillator in a low-noise Double-Balanced Mixer (DBM). The low-pass fil- 


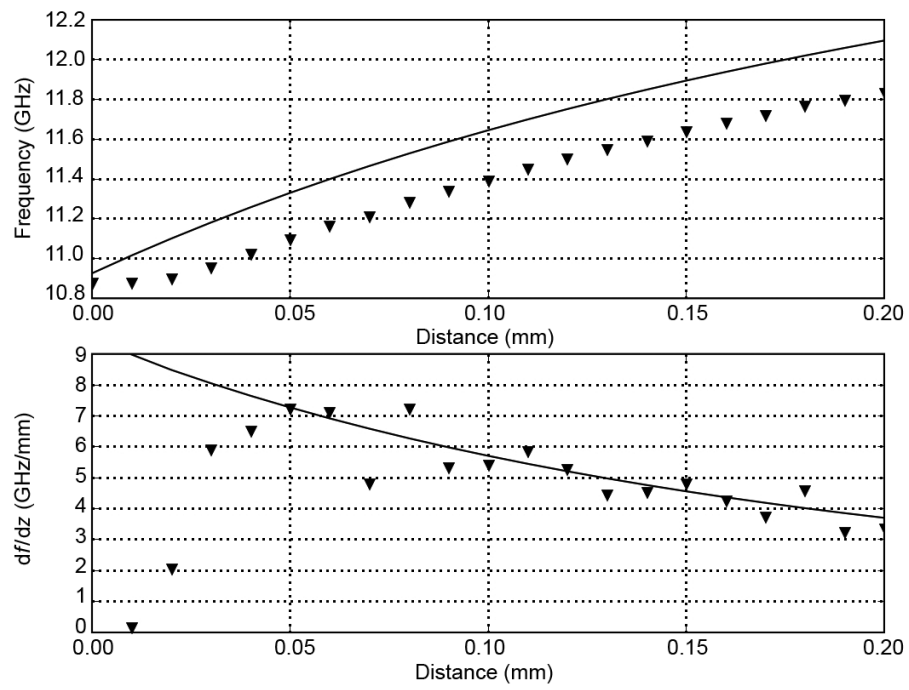

Fig. 6. Resonance frequency and tuning coefficient of the $E_{11,1,1}$ mode in function of the distance between the two disk of $42 \mathrm{~mm}$ diameter and $5 \mathrm{~mm}$ height (points-experimental values; line-theoretical values).

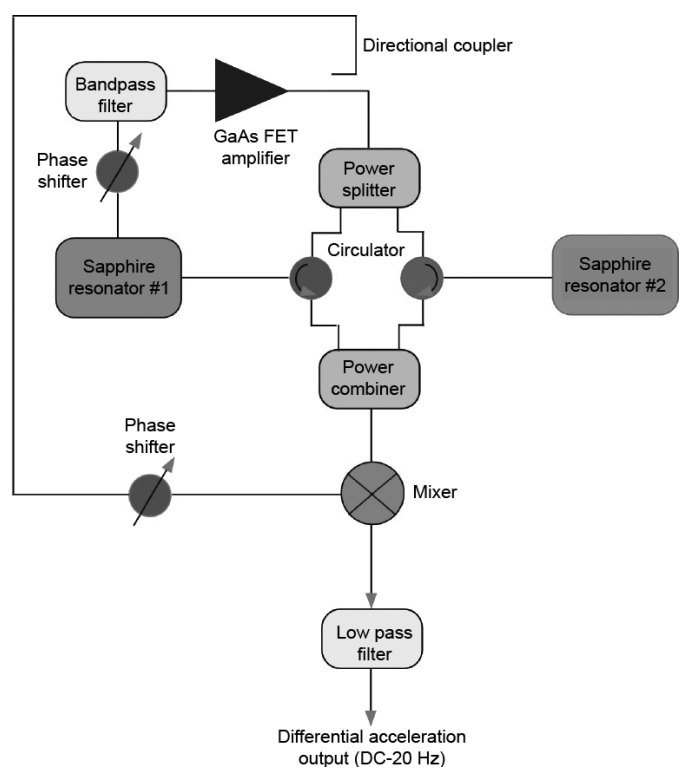

Fig. 7. Schematic of the interferometric microwave readout system. One resonator is used as the reference cavity for a $X$-band loop-oscillator. Its output is used to interrogate the other dielectric resonator and the frequency difference is read by a double-balanced mixer. tered output of the DBM is a voltage proportional to the sensed differential acceleration, in the frequency band DC-20 Hz. The balanced interferometric scheme allows for carrier suppression, enhancing the resolution, especially at low frequencies. Otherwise the sensitivity would be limited by the loop oscillator phase noise and by the frequency discriminator noise. By considering a reasonable dynamic range of $130 \mathrm{~dB}$ for the mixer, the frequency mismatch between the two microwave resonators must be limited within $100 \mathrm{kHz}$ to allow the gradiometer to operate. A trimming procedure will be then necessary, after the machining, to suppress the bias effect due to shape mismatch in the dielectric resonators, producing a static frequency offset. The high mechanical $Q$ level calls for a mechanism that limits the dynamics of the oscillators. This will be done by an active damping feedback control as used in sensitive detectors like the VIRGO gravitational antenna (Acernese et al., 2004). Each sensor will be fitted with an actuator consisting in a small magnet fixed on the upper face of the moving arm, driven by a coil fixed to the frame. A laser beam is reflected by the moving arm and sent to a position sensitive detector. A current signal propor- 
tional to the moving arm velocity is injected. It is possible in this way to reduce the dynamic of the oscillation by orders of magnitude.

The minimum detectable gravity gradient per bandwidth unit can be evaluated by comparing the expected signal with the noise. By considering thermal noise due to the Brownian motion of the accelerometer test masses and the phase noise of the loop oscillator, we find the equation

$$
\begin{aligned}
& \Gamma(\omega)=\frac{\omega_{0}^{2}}{l} . \\
& \sqrt{\left(\frac{\omega}{\pi \frac{\mathrm{d} f}{\mathrm{~d} x}}\right)^{2} \varepsilon^{2} S_{\phi}(\omega)+\frac{4 k_{B} T \omega_{0}}{m Q\left[\left(\omega^{2}-\omega_{0}^{2}\right)^{2}+\left(\omega \omega_{0} / Q\right)^{2}\right]}}
\end{aligned}
$$

where $\omega_{0}$ is the mechanical resonant frequency, $l$ is the accelerometer baseline, $m$ is the test mass, $Q$ is the mechanical quality factor, $\mathrm{d} f / \mathrm{d} x$ is the tuning coefficient, $S_{\phi}$ is the loop oscillator phase noise spectral density and $\varepsilon$ is the balancing factor of the interferometric system. At room temperature typical expected parameters (Tobar et al., 1993) are $Q \approx 10^{4}, S_{\phi} \approx 10^{-3} / f^{2} \mathrm{rad}^{2} / \mathrm{Hz}$ and $\varepsilon \approx$ $\approx 0.01$, yielding $\Gamma_{\min } \approx 1$ Eö for 1 -s of integration time.

\section{Conclusions}

We have presented the design features of the core differential accelerometer for new low cost lightweight gravity gradiometer instruments suitable for land geophysical surveys. The combination of the monolithic CNC machined mechanics and of a fine electrostatic stiffness balancing would produce a very high mechanical CMRR, of the order of $120 \mathrm{~dB}$, allowing 1-Eö level resolutions to be achieved.

At present the main components of the first prototype are being developed and fixed. The core mechanics is being built in the ultrasonic machining facility of University of Pisa, using a low cost, lower purity grade HEMLITE ${ }^{\mathrm{TM}}$ sapphire. The microwave readout system design is being done by investigating on the WG modes of the single transducer. After the integration of the core sensor with the necessary sub-systems like the temperature control, the gimbal leveling frame, the balancing jig etc., a first labora- tory prototype of $\Gamma_{y z}$ gradiometer will be tested. Moreover new $\Gamma_{z z}$ sensors, less sensitive to the alignment with respect to the vertical direction, will be designed.

\section{Acknowledgements}

This work has been partially supported by «Fondazione della Cassa di Risparmio di Pisa».

\section{REFERENCES}

ACERnESE, F. et al. (2004): A local control system for the test masses of the VIRGO gravitational wave detector, Astroparticle Phys., 20, 617-628.

Bertolini, A., N. Beverini, G. Dal Lago, R. Desalvo, F. Fidecaro, F. Francesconi, M. Francesconi and G. OMiCCIOLI (2003): A 5-axis CNC ultrasonic cutting machine: design and preliminary test, LIGO Technical Note T020198-00.

Blair, D.G., E.N. Ivanov and H. Peng (1992): Sapphire dielectric resonator transducers, J. Phys. D, 25, 1110.

Blair, D.G., E.N. Ivanov and H. Peng (1994): An ultrahigh sensitivity sapphire transducer for vibration measurements, J. Phys. D, 27, 1150.

Blair, D.G., E.N. Ivanov, M.E. Tobar and B.D. CuthberTSON (1998): Sensitivity and optimization of a high-Q sapphire dielectric motion-sensing transducer, IEEE Trans. Ultrason. Ferroelect. Freq. Contr., 45, 1303-1313.

ChaIn, H.A. and H.J. PAIK (1988a): Superconductive gravity gradiometer for sensitive gravity measurements, I. Theory, Phys. Rev. D, 35, 3551 .

Chain, H.A. and H.J. PAIK (1988b): Superconductive gravity gradiometer for sensitive gravity measurements, II. Experiment, Phys. Rev. D, 35, 3572.

Goldstein, M.S. and J.J. BRETT (1998): Precision gravity gradiometer/AUV system, in Proceedings of the 1998 Workshop on Autonomous Underwater Vehicles, AUV'98, 167-174.

LEE, J.B. (2001): FALCON gravity gradiometer technology, Exploration Geophys., 32, 247-251 (available on line at http://falcon.bhpbilliton.com/docs/falcon_gravity_gradiometer_technology.pdf).

Matthews, R. (2002): Mobile Gravity Gradiometry, PhD Thesis (University of Western Australia), (unpublished).

Rebhan, H., M. Aguirre and J. Johannessen (2000): The gravity field and steady-state ocean explorer mission GOCE, ESA Earth Observation Q., 66, 6-11.

RichteR, B. and R.J. WARBURTON (1998): A new generation of superconducting gravimeters, in Proceedings of the 13th International Symposium on Earth Tides, Série Géophysique, Royal Observatory of Belgium, Brussel, 545-556.

SnAdDEN, M.J., J.M. McGuiRK, P. Bouyer, K.G. Haritos and M.A. KASEVICH (1998): Measurement of the Earth's gravity gradient with an atom interferometer-based gravity gradiometer, Phys. Rev. Lett., 81, 971-974.

Tobar, M.E., A.J. GiLES, S. EdwARdS and J. SEARLS (1993): 
High $Q$ TE stabilised sapphire microwave resonators for low noise applications, in Proc. IEEE Frequency Control Symposium, U.S.A.

Van Kann, F.J., M.J. Buckingham, C. Edwards and R. MATTHEws (1994): Performances of a superconducting gravity gradiometer, Physica B, 194 and 196, 61-62.

(received January 28, 2006; accepted July 20, 2006) 